\title{
A decision support tool for supporting individuals living with long-term conditions make informed choices: LTC-Choices tool for continuous healthcare
}

Julie Cowie ${ }^{\mathrm{a} *}$ and Frada Burstein ${ }^{\mathrm{b}}$

${ }^{a}$ Faculty of Health Sciences and Sport, University of Stirling, Stirling, Scotland, UK

${ }^{\mathrm{b}}$ Centre for Organisational and Social Informatics, Faculty of Information Technology, Monash University, Melbourne, Australia

julie.cowie@stir.ac.uk, frada.burstein@monash.edu

This is an Accepted Manuscript of an article published by Taylor \& Francis Group in Journal of Decision Systems on 16 Apr 2018, available online: http://www.tandfonline.com/10.1080/12460125.2018.1460157 


\title{
A decision support tool for supporting individuals living with long-term conditions make informed choices: LTC-Choices tool for continuous healthcare
}

\begin{abstract}
An increasing number of individuals are now living with some form of chronic, long-term condition (LTC). The holistic perspective of LTCs makes it important to acknowledge that priorities and decisions are in fluctuation over the course of an individual's life. The landscape of digital healthcare is full of information systems that capture individuals' health data, clinical guidelines and/or advice on health conditions, which taken together can help create a comprehensive overview of suitable lifestyle choices to optimise health and well-being. Despite this, there is no evidence of existing frameworks to support individuals living with LTCs from a continuum of care perspective. In this paper we propose such a multidimensional model for a decision support tool - LTC-Choices. This tool was developed from existing work conducted by the authors around use of multicriteria to support health decision making. We illustrate how LTC-Choices can be implemented using the example of individuals living post-stroke.
\end{abstract}

Keywords: multicriteria, decision making, long term condition, digital health, stroke

\section{Introduction}

The number of individuals living with long-term conditions (LTCs) globally is increasing (Department of Health, 2012). The size of the chronic disease population, particularly those with multiple comorbidities, is projected to grow, reaching 157 million Americans by 2020 (Kane et al., 2005, p212). In the UK, around 6 out of 10 people surveyed in 2001 and 2002 reported some sort of chronic problems (Wilson et al., 2005). In Australia chronic conditions are responsible for around $80 \%$ of disability affected people (NHPAC, 2006). Care of those with long-term conditions puts huge demands and increases financial pressure on services (Jones et al., 2011).

Promoting self-management amongst those living with LTCs is believed to provide benefits to individuals by increasing power and responsibility over treatment process and 
promoting autonomy (Lenzen et al., 2015). To date, clinical decision support aids that embed some form of interactive decision support model/element have only been used in a clinical setting under the guise of promoting shared decision making (Lawn et al., 2011). Tools for individuals to use at home, in the community, to self-manage, mainly focus on information provision and communication as a means of decision support. Should the individual need/want to make a decision about an aspect of their condition/care, there is no guidance as to how to use such information to make an informed choice.

In this paper we propose a decision support tool for use by individuals living with a long-term condition. The tool - LTC-Choices - does not focus on making short-term decisions, rather it aims to help the individual assess their condition from a long-term goal setting perspective. LTC-Choices is developed around a multivariate decision model (Belton and Stewart, 2002) and is informed by the factors recognised in clinical guidelines (for example, the National Institute for Health and Care Excellence guidelines (2018) and relevant clinical literature available on specific long-term conditions (for example, SIGN (2010)). We present the rationale for the development of LTC-Choices and discuss its structure and design. The potential of the tool is illustrated using the example of supporting people living post-stroke. Future work will include a field-test of the proposed architecture with practitioners and the stroke-survivors community, employing a co-production model to ensure it is fit for purpose.

\section{Self-management for those with long term conditions}

Strategy documents such as the Modern Outpatient (Scottish Government, 2016) and others (for example, Queensland Government, 2016), advocate for a new model in care provision, where the patient is supported to (where appropriate) take responsibility for their health and wellbeing. Such a shift in the balance of care attempts to promote wellness and 
in turn, reduce interactions with health care services. In line with this approach, selfmanagement is seen as a means of increasing the capacity, confidence, and efficacy of an individual (NHS England, 2017). Amongst those living with a long-term condition, selfmanagement can also provide a sense of control, promote autonomy and give individuals a sense of greater responsibility over treatment process (Lenzen et al., 2015).

Dwardswaard et al. (2015) separate self-management for those with LTCs into two overarching areas: disease related self-management and individual self-management. Their view is that self-management behaviour can be very dependent on the disease stage. For example, at diagnosis or soon after, patients are concerned with information about the disease, its profile and available treatments and deciding between these. In the chronic phase of the disease, focus may move towards psychological support, and patients who feel capable of self-management may only require support should their condition worsen. Supporting the individual self-manage at this stage in a disease profile can relate to factors around lifestyle, life circumstances and how these can impact on their sense of coping (van Eijk-Hustings et al., 2013; Dwarswaard et al., 2015). This view of self-management encompassing both condition specific and life style/circumstances is well-regarded and widely recognised (Haggerty et al, 2003).

\section{Role of eHealth in self-management of long term conditions}

There are many ways in which individuals can be encouraged to self-manage. One area that has received much attention is that of eHealth, particularly in relation to use of websites and/or apps providing information on aspects such as illness trajectories, symptoms, and treatments relating to specific conditions (Wong \& Lam, 2016; Con et al., 2017). Although potentially effective, patient-based interventions such as eHealth are still currently considered limited in their "reach", and adoption of such systems still in its 
infancy. Concerns are often raised about use of such systems citing that information can be misinterpreted and misused. Reasons for this include inadequate health literacy (Gutierrez et al, 2014) or an individual's inability to synthesize such vast amounts of health related information (Leroy et al., 2013). These are in addition, to concerns raised about the accuracy of information provided, where patients have free access to non-accredited apps (Eysenbach, 2014).

Such factors are used by some to advocate for decisions to be made in conjunction with a health care professional, who can support patients in their decision making and ensure their understanding and use of the information is appropriate. However, there is recognition that there are limitations in providing shared decision-making as clinicians are so time poor and often cannot devote adequate time to ensuring shared decision-making takes place (Dwarswaard et al., 2015). O'Grady \& Jadad (2010) advocate for the need to shift from shared to collaborative decision making to improve patient care. They believe that in order to achieve such a shift, patients need to acquire relevant knowledge about their condition and gain confidence to engage in better understanding the life choices they have. We support their view and highlight the need for decision support tools which help guide the individual in their decision and direct them to information appropriate to their needs. As highlighted, for those with LTCs, decision-making is a life-long exercise which is multi-faceted and changeable. Use of a tool to support such individuals make decisions and set goals as and when they want is seen to provide greater empowerment and control over life choices.

\section{LTC-Choices: Supporting decisions throughout the continuum of care}

LTC-Choices is a proof-of-concept tool designed to support individuals with LTCs make informed choices as part of their continuum of care. The tool supports holistic decision 
making that is personalised and adaptive to facilitate the changing needs of individuals as they live life with their condition.

\section{LTC-Choices Dashboard}

Health informatics literature identifies multiple information sources which provide the basis for life choice medical decision making (O’Grady \& Jadad, 2010). In figure 1 we propose an overall architecture of the LTC-Choices tool, which integrates information sources derived from clinical records and medical knowledge-bases (Clinical information) and information around an individual's lifestyle and other contextual factors, which individuals need to take into account when making decisions about their treatment and life style choices.

Figure 1. LTC-Choices Architecture

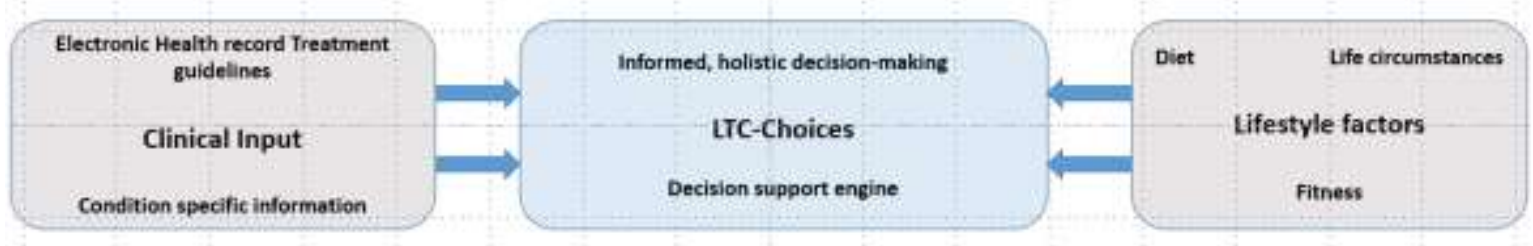

By way of example, in this figure we suggest Clinical Input might be derived from a patient's electronic health record (such as myHealth record, for example, as adopted in Australia, and other countries (Adler-Milstein et al, 2014)), or perhaps standard quality controlled clinical guidelines (such as those provide by NICE in the UK (NICE guidelines (2018)). Lifestyle factors could include aspects such as information about the individual's fitness, current diet, and life circumstances (which could include an array of information relating to aspects such as whether the individual lives alone, social networks, support networks, dependents).

To demonstrate how the LTC-Choices infrastructure might be developed, we use the example of stroke. The authoritative source of clinical factors might be derived from 
medical knowledge bases such as the guidelines provided by the National Heart Foundation of Australia \& Cardiac Society of Australia and New Zealand (Chew et al, 2016), or in the UK corresponding NICE Guidelines around stroke (NICE, 2013).

Although these sources are authoritative and highly comprehensive, the information provided is mainly directed towards clinical professionals. However, these sources can illuminate key clinical aspects to incorporate in a decision support tool. With regard to lifestyle decisions, through collaboration with patient communities, carer groups and consulting specialists in lifestyle decisions, a list of factors which contribute to improved health outcomes can be identified and presented to the users to guide their selection of a profile which is the closest to their own circumstances.

\section{Decision support engine}

In order to assimilate information from both clinical and lifestyle sources, an appropriate means of structuring the information in a meaningful way is an essential component of LTC-Choices. Multicriteria decision analysis (MCDA) is a decision making model that has been employed in a variety of applications, notably in financial investment planning (Zopounidis \& Doumpos, 2002) and health (Marsh et al. (2016), Angelis \& Kanavos (2017)) .Within the MCDA field, there are many different formal approaches that have been developed with the aim of ensuring a structured approach to decision making (Belton \& Stewart, 2002). We have adopted the Multiattribute value function (MAVT) approach as this is empirically well validated (Belton, 1990; French et al., 1998) and has been widely used in health decision making (Angelis \& Kanavos, 2017; Dolan, 2010). The two main components of the value function are the evaluation scores of alternatives with respect to criteria, and the relative importance (or weight) of each criterion. 


\section{Example application - life after stroke}

In order to demonstrate how LTC-Choices might support decisions in practice, we present the tool using the example of stroke. For the purpose of analysis, we have referred to the health care systems in both the UK and Australia as these are the health systems the authors are most familiar with. In addition, in a study commissioned by the Commonwealth Fund, these two health systems were found to be the highest performing health care systems worldwide (Schneider et al, 2017).

In the UK there are currently over 1.2 million stroke survivors (Stroke Association, 2017) and in Australia 475,000, which is likely to increase to 1 million by 2020 (Stroke Foundation, n.d.). Of these, almost 2/3rds of survivors face life after stroke with a disability. Both in the UK and Australia, there are excellent resources supporting individuals living with life after stroke. These include a wide array of support groups as well as detailed websites providing excellent information resources. For example, in Australia, the Stroke Foundation provides a website EnableMe (EnableMe, 2018) which provides a wealth of information about life after stroke. Similarly, in the UK, the Stroke Association website (Stroke Association, n.d.) provides information on life after stroke, as well as information provision around possible problems individuals may face after suffering a stroke. We regard LTC-Choices as a tool that complements these information resources in a way that allows individuals living post-stroke to make use of such information to make informed decisions about their life and/or treatment options they might be considering.

\section{LTC-Choices Stroke MCDA model}

In figure 2, we propose a post-stroke decision support model - STROKE-Choices. The model incorporates recognised factors that can occur/be of concern to individuals who 
have suffered a stroke (Jaracz \& Kozubski, 2003; Cunningham et al, 2017). It incorporates Flanagan's Quality of life scale (Flanaghan, 1978) as depicted by the criteria listed under the Quality of life scale criterion. Flanagan's scale is widely recognised for its reliability, validity and suitability in measuring quality of life across patient groups and cultures (Burckhardt et al., 2003). Criteria listed under the Problems experienced criterion are those listed on the UK's Stroke association website (Stroke association, n.d.) as known problems those living post-stroke may experience. Care burden is included as a criterion as it is a recognised factor that individuals take into account when assessing life choices after having suffered from a stroke (Cunningham et al, 2017).

In this paper, we only discuss the STROKE-Choices decision engine. Through employment of a co-production model with stroke survivors, current work is focused on the STROKE-Choices interface. It is anticipated that the tool will comprise of a userfriendly front-end which, through a series of questions to the user, would populate the back-end STROKE-Choices MCDA model in order to determine areas the individual may wish to address. An example scenario of its use might be that of an individual living poststroke who wants to decide what their lifestyle priorities are for the next week/month/year/long-term. This could include aspects such as trying to regain mobility, being less-socially isolated, or perhaps finding a new hobby to engage in. Working through the series of questions they are asked using the STROKE-Choices tool would allow the individual to identify areas to focus on. Questions are directly linked to the criteria incorporated in the STROKE-Choices MCDA model. For each criterion, the individual is asked how well they are doing with that aspect of their life and how important it is to them. 


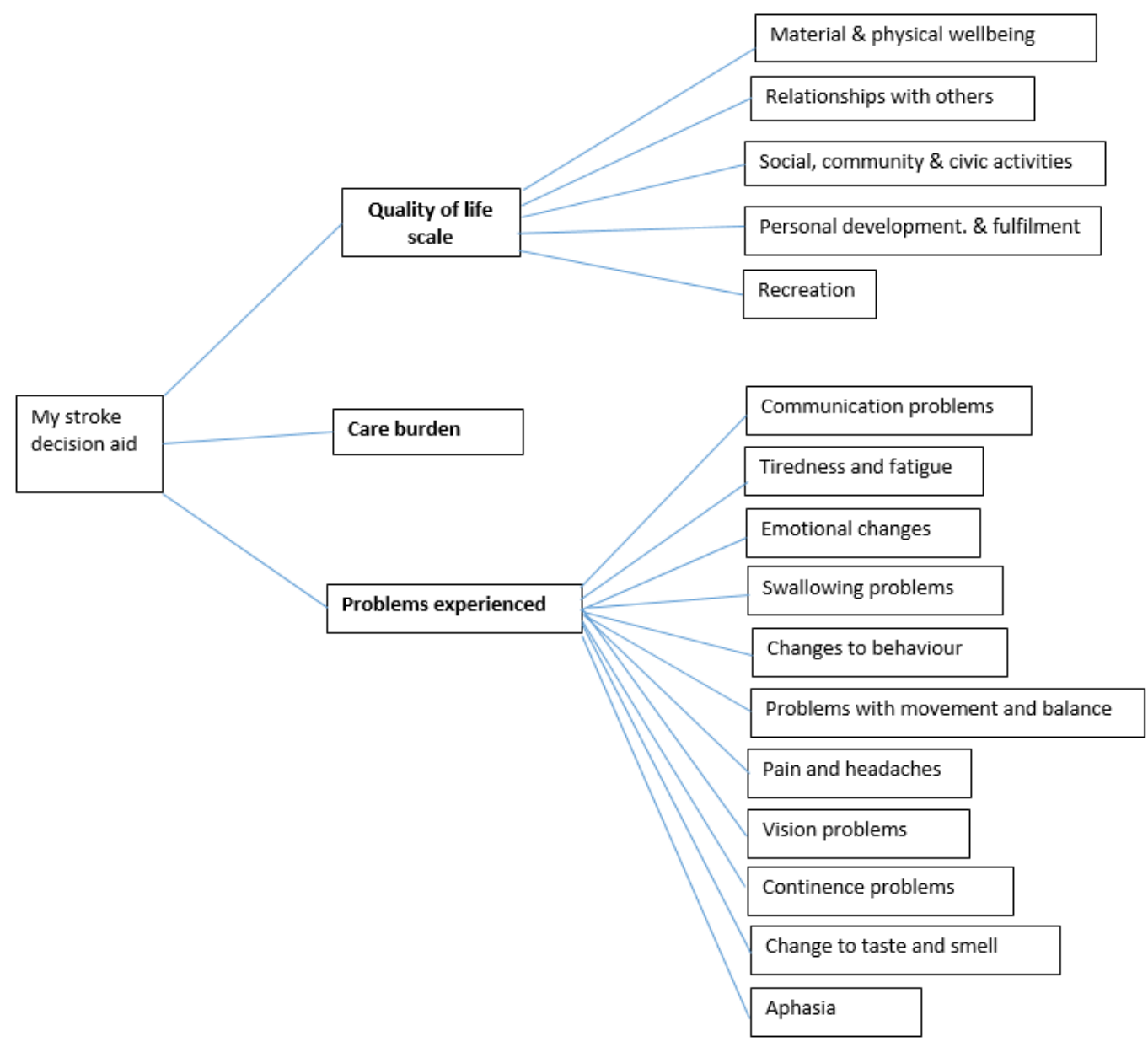

So for example, STROKE-Choices may ask the individual "How socially connected are you (for example, do you attend social events, are you part of a community group)?" This question would provide a score for the "Social, community and civic activities" criterion. A second question would be "How important to you is feeling socially connected?" This question allows the individual to express the significance of this aspect of their life. An example scenario might be the individual giving a low score on this criterion (don't currently feel social connected) accompanied by a high weight (it is important to them) which would indicate being socially connected is an area that the individual regards as important to them, but that they don't feel they currently achieve in their life. 


\section{Discussion and future work}

Credible, quality information is critical for anyone making decisions regarding their health (Nguyen, et al., 2015). In this paper we explore a proof-of-concept around the provision of a decision support tool to support those living with long term conditions making informed choices. We recognise the continuum of care that such individuals require and that decision making is not a one off event, but something that such individuals face throughout life, sometimes on a daily basis. LTC-Choices can provide a means by which information can be assimilated to support individuals decide their priorities and goals in relation to their current health and wellbeing. Such structured, informed decision making we believe can support individuals in goal-setting by recognising what aspects of their current health and well-being they want to change.

In order to determine whether LTC-Choices is a concept that individuals with LTCs would find useful, we are currently conducting a series of focus groups with individuals and carers of individuals living beyond stroke. In addition, we will be facilitating a number of meetings with clinicians to assess the tool's potential. The data from these workshops and interviews will be used to inform the next stage of our work, which will focus on the development of a prototype LTC-Choices and its subsequent evaluation.

\section{Shifting the balance of care}

As intimated earlier, clinical decision making is often regarded as an activity that takes place between patient and health care professional. In line with strategies around empowering individuals to take responsibility for their own care and make informed choices we feel LTC-Choices can facilitate this shift in care provision through helping the individual make more informed choices. However, we also recognise that despite strategic documents, the culture around care provision needs to change: Patient and clinician 
education and engagement in shifting the balance of care such that the individual takes

ownership for their care where appropriate is essential if tools such as LTC-Choices are to be successfully implemented and integrated into care provision (Coulter, 2011).

\section{References}

Adler-Milstein, J., Sarma, N., Woskie, L. R., \& Jha, A. K. (2014). A comparison of how four countries use health IT to support care for people with chronic conditions. Health Affairs, 33(9), 1559-1566.

Angelis, A. \& Kanavos, P. (2017). Multiple Criteria Decision Analysis (MCDA) for evaluating new medicines in Health Technology Assessment and beyond: The Advance Value Framework. Social Science \& Medicine, 188, 137-156.

Barnett, K., Mercer, S.W., Norbury, M., Watt, G., Wyke, S., \& Guthrie, B. (2012). Epidemiology of multi-morbidity and implications for health care, research, and medical education: a cross-sectional study. Lancet, 380(9836), 37-43.

Belton, V. (1990). Multiple Criteria Decision Analysis - Practically the Only Way to Choose. In Hendry L.C. and Eglese R.W. (Eds) Operational Research Tutorial Papers, Operational Research Society, Birmingham, 53-101.

Belton, V. \& Stewart, T.J. (2002). Multiple Criteria Decision Analysis: An Integrated Approach. Springer US.

Bodenheimer, T., \& Handley, M.A. (2009). Goal-setting for behavior change in primary care: An exploration and status report. Patient Education Couns., 76, 174-80.

Bodenheimer, T., \& Grumbach, K. (2007). Self-management support for people with chronic illness. In: Shanahan, J.F., Brown, R. (Eds) Improving primary care: strategies and tools for a better practice. 1st ed. New York: The MacGraw-Hill Companies.

Burckhardt, C.S., Anderson, K.L., Archenholtz, B. \& Hägg, O. (2003). The Flanagan Quality of Life Scale: Evidence of Construct Validity. Health Quality Life Outcomes. 2003(1), 59.

Chew, D.P., Scott, I.A., Cullen, L., French, J.K., Briffa, T.G., Tideman, P.A., Woodruffe, S., Kerr, A., Branagan, M. and Aylward, P.E., 2016. National Heart Foundation of Australia \& Cardiac Society of Australia and New Zealand: Australian clinical guidelines for the management of acute coronary syndromes 2016. Heart, Lung and Circulation, 25(9), 895-951.

Con, D., Jackson, B., Gray, K. \& De Cruz, P. (2017). eHealth for inflammatory bowel disease self-management - the patient perspective. Scandinavian Journal of Gastroenterology. Retrieved from https://www.tandfonline.com/doi/abs/10.1080/00365521.2017.1333625?journalCo de=igas 20

Coulter A. (2011). Engaging patients in healthcare. New York: Open University Press.

Cunningham, N., Abhyankar, P., Cowie, J., Galinsky, J. \& Methven, K. (2017). Regenerative Medicine: Stroke survivor and carer views and motivations towards a proposed stem cell clinical trial using placebo neuro-surgery Health Expectations 2017, 1-17.

Department of Health. (2012). Long-term conditions compendium of Information: $3 \mathrm{rd}$ edition. Retrieved from 
https://www.gov.uk/government/uploads/system/uploads/attachment_data/file/2165 28/dh_134486.pdf

Dolan, J.G. (2010). Multi-Criteria Clinical Decision Support. The Patient: Patient-Centred Outcomes Research, 3(4), 229-248.

Dwarswaard, J., Bakker, E.J.M., van Staa, A., Boeije, H.R. (2015). Self-management support from the perspective of patients with a chronic condition: a thematic synthesis of qualitative studies. Health Expectations, 19(2), 194-208.

EnableMe. (2018). Retrieved from https://enableme.org.au/

Eysenbach, G. (2014). The new health-related top-level domains are coming: will cureforcancer.health go to the highest bidder? J Med Internet Res.,16, 3, e73.

Flanagan, J.C. (1978). A research approach to improving our quality of life. Am Psychologist, 33:138-147.

French, S., Simpson, L., Atherton, E., Belton, V., Dawes, R., Edwards, W., Hämäläinen, R., Larichev,O., Lootsma, F., Pearman A., and Vlek, C. (1998) 'Problem formulation for multi-criteria decision analysis: report of a workshop' J. MultiCriteria Decision Analysis, 7, 242-262.

Gutierrez, N., Kindratt, T.B., Pagels, P., Foster, B., \& Gimpel, N.E. (2014). Health literacy, health information seeking behaviors and internet use among patients attending a private and public clinic in the same geographic area. $J$ Community Health. 39(1), 83-9.

Haggerty, J.L., Reid, R.J., Freeman, G.K., Starfield, B.H., Adair, C.E., \& McKendry, R. (2003). Continuity of care: a multidisciplinary review. BMJ, 327, 1219-1221.

Jaracz, K. \& Kozubski, W. (2003). Quality of life in stroke patients. Acta Neurologica, 107 (5), 324-329.

Jones, M.C., MacGillivray, S., Kroll, T., Zohoor, A.R., \& Connaghan, J. (2011). A thematic analysis of the conceptualisation of self-care, self-management and selfmanagement support in the long-term conditions management literature. J Nurs Healthc Chronic Illn., 3, 174-85.

Kane, R.L., Priester, R., Totten, A.M. (2005). Meeting the Challenge of Chronic Illness. The Johns Hopkins University Press.

Lawn S, \& Schoo A. (2010). Supporting self-management of chronic health conditions: Common approaches. Patient Educ Couns., 80, 205-11.

Lawn, S., McMillan, J., \& Pulvirenti, M. (2011). Chronic condition self-management: expectations of responsibility. Patient education and counseling, 84(2), e5-e8.

Lenzen, S. A., Daniëls, R., van Bokhoven, M. A., van der Weijden, T., \& Beurskens, A. (2015). Setting goals in chronic care: Shared decision making as self-management support by the family physician. European Journal of General Practice, 21(2), 1-7.

Leroy, G., Endicott, J., Kauchak, D., Mouradi, O., Just, M. (2013). User evaluation of the effects of a text simplification algorithm using term familiarity on perception, understanding, learning, and information retention. J Med Internet Res., 15(7), e144.

Marsh, K., Ijzerman, M., Thokala, P., Baltussen, R., Boysen, M., Lalo, Z.,...Devlin, N. (2016) Multiple Criteria Decision Analysis for Health Care Decision Making Emerging Good Practices: Report 2 of the ISPOR MCDA Emerging Good Practices Task Force. Value in health 19. Retrieved from https://www.ispor.org/Multi-Criteria-Decision-Analysis-guideline-2.pdf

National Health Priority Action Council (NHPAC) (2006). National Chronic. Disease Strategy, Australian Government Department of Health and Ageing, Canberra. Retrieved from https://extranet.who.int/ncdccs/Data/AUS_B3_Att\%20A\%20- 
\%20National\%20Chronic\%20Disease\%20Strategy\%202005\%20(D15-

706588).PDF

National Institute for Health and Care Excellence. (2018). NICE Guidelines. Retrieved from https://www.nice.org.uk/about/what-we-do/our-programmes/niceguidance/nice-guidelines

National Institute for Health and Care Excellence. (2013). NICE Guidelines. Stroke Rehabilitation in Adults. Retrieved from https://www.nice.org.uk/guidance/cg162/resources/stroke-rehabilitation-in-adultspdf-35109688408261

Nguyen, B., Burstein, F., \& Fisher, J (2015). Improving service of online health information provision: A case of usage-driven design for health information portals. Information Systems Frontiers, 17(3), 493-511.

NHS England (2017). Involving people in their own health and care: statutory guidance for clinical commissioning groups and NHS England. Retrieved from https://www.england.nhs.uk/wp-content/uploads/2017/04/ppp-involving-peoplehealth-care-guidance.pdf

O'Grady, L., \& Jadad, A. (2010). Shifting from shared to collaborative decision making: a change in thinking and doing. Journal of Participatory Medicine, 2(13), 1-6.

Queensland Goverment (2016). Specialist Outpatient Strategy. Improving the patient journal by 2020. Retrieved from https://www.health.qld.gov.au/_data/assets/pdf_file/0027/443934/specialistoutpatient-strategy.pdf

Schneider, E.D., Sarnak, D.O., Squires, D., Shah, A., \& Doty, M.M. (2017). Mirror, Mirror 2017: International Comparison Reflects Flaws and Opportunities for Better U.S. health Care. The Commonwealth Fund. Retrieved from http://www.commonwealthfund.org/interactives/2017/july/mirror-mirror/

Scottish Goverment (2016). The Modern Outpatient: A Collaborative Approach 20172020. Retrieved from http://www.gov.scot/Resource/0051/00510930.pdf

Scottish Intercollegiate Guidelines Network (SIGN). (2010). Management of patients with stroke: Rehabilitation, prevention and management of complications, and discharge planning. A national clinical guideline. Retrieved from http://www.sign.ac.uk/assets/sign118.pdf

Stroke Association (2017) State of the nation, stroke statistics. Retrieved from https://www.stroke.org.uk/sites/default/files/state_of the nation_2017_final_1.pdf

Stroke Association (n.d.) Retrieved from https://www.stroke.org.uk/

Stroke Foundation (n.d.) Retrieved from https://strokefoundation.org.au/

Van eijk-Hustings, Y., Ammerlaan, J., Voorneveld-Nieuwenhuis, H., Maat, B., Veldhuizen, C., \& Repping-Wuts, H. (2013). Patients' needs and expectations with regard to rheumatology nursing care: Results of multicentre focus group interviews. Annals of the Rheumatic Diseases, 72, 831-835.

Wilson, T., Buck, D., \& Ham, C. (2005). Rising to the challenge: will the NHS support people with long term conditions? BMJ : British Medical Journal, 330(7492), 657661.

Wong, M.L., \& Lam, G.Y.Y. (2016). Can E-Health Support Programme Improve SelfManagement for Diabetes Patients in the Community? Journal of Community \& Public Health Nursing. Retrieved from https://www.omicsonline.org/openaccess/can-ehealth-support-programme-improve-selfmanagement-fordiabetespatients-in-the-community-jcphn-1000e111.pdf 
Zopounidis, C., \& Doumpos, M. (2002). Multi-criteria decision aid in financial decision making: methodologies and literature review. Journal of Multi-Criteria Decision Analysis, 11(4-5), 167-186. 